\title{
Curcuma longa Aided Ag/CS Nanocomposite Coating of Surfaces as SARS-CoV-2 Contamination Minimizing Measure Towards Containment of COVID-19: a Perspective
}

\author{
Poushpi Dwivedi ${ }^{1, *}$ (D), Dhanesh Tiwary ${ }^{1}$, Shahid S. Narvi ${ }^{2}$, Ravi P. Tewari ${ }^{3}$, Keshav P. Shukla ${ }^{4}$ \\ 1 Department of Chemistry, Indian Institute of Technology (Banaras Hindu University), Varanasi, UP, India 221005 \\ 2 Chemistry Department, Motilal Nehru National Institute of Technology Allahabad, UP, India 211002 \\ 3 Applied Mechanics Department, Motilal Nehru National Institute of Technology Allahabad, UP, India 211002 \\ 4 Biotechnology Department, Motilal Nehru National Institute of Technology Allahabad, UP, India 211002 \\ * Correspondence: poushpid.pf.chy17@itbhu.ac.in;
}

Scopus Author ID 54896254200

Received: 14.07.2020; Revised: 11.08.2020; Accepted: 12.08.2020; Published: 15.08.2020

\begin{abstract}
In the ongoing pandemic outbreak of the coronavirus disease 2019 caused by Severe Acute Respiratory Syndrome Coronavirus 2, rapid research promoting irradiation strategies to approved therapeutics and authorized immunity-boosting options for eradication of the novel coronavirus is still at a primary stage. Based on the evidence, a perspective of contamination minimizing measure is presented here, providing preventive technique through a maximized coating of surfaces with selfsterilizing antimicrobial nanocomposite material, consisting of antiviral properties to effectively combat the threat. Curcuma longa $\mathrm{L}$. and polyphenolic compound curcumin present in its rhizome, since ages have been subjected to extensive antimicrobial investigations and exhibit proven antiviral activity, possessing synergism enhancement characteristic to the inhibitory effect of other antimicrobial agents. Silver nanoparticles, also considered potential tool against a variety of viruses and effectiveness of the silver/chitosan coating system is amplified in combination with adherent curcumin from Curcuma longa rhizome extract, which effectuated nanosilver generation for the approach. Nanobiotechnological synthesis of silver/chitosan nanocomposite antimicrobial surface coating, aided by Curcuma longa rhizome, chiefly consisting of curcumin. Synergistic effect of curcumin and nanosilver antiviral agents minimizing contamination. Facile coating system feasible over maximum exposed surrounding contact areas having metallic, ceramic, polymeric, wooden, etc., surface texture. The self-sterilization and unchanged function of commonly handled handles, railings, switches, touch screens, keyboards, tabletops, etc. They are imparting preventive measures to combat SARS-CoV-2 associated COVID-19 infection.
\end{abstract}

Keywords: Silver nanocomposite; Curcumin; Antiviral; Surface coating; SARS-CoV-2; COVID-19.

(C) 2020 by the authors. This article is an open-access article distributed under the terms and conditions of the Creative Commons Attribution (CC BY) license (https://creativecommons.org/licenses/by/4.0/).

\section{Introduction}

Despite drastic containment actions adapted globally, towards the dreaded pandemic of the coronavirus disease 2019 (COVID-19), posed by the novel coronavirus, officially labeled as Severe Acute Respiratory Syndrome Coronavirus 2 (SARS-CoV-2), the results are far from satisfactory and this pneumonia-like extremely contagious disease is spreading fast vulnerably [1]. SARS-CoV-2 targets angiotensin-converting enzyme 2 (ACE2), which is a receptor 
expressed in the human lungs, gastrointestinal tract, cardiovascular, and central nervous systems [2, 3]. Coronaviruses are single-stranded RNA viruses having a diameter of $80-120 \mathrm{~nm}$. They are divided into four kinds: alpha-coronavirus $(\alpha-\mathrm{CoV})$, beta-coronavirus $(\beta-\mathrm{CoV})$, deltacoronavirus $(\delta-\mathrm{CoV})$ and gamma-coronavirus $(\gamma-\mathrm{CoV})[1]$. Previously, six types of coronaviruses were known to affect humans, causing diseases, SARS-CoV-2 being the seventh variety of the coronavirus family, after Severe Acute Respiratory Syndrome Coronavirus 1 (SARS-CoV) and the Middle East Respiratory Syndrome Coronavirus (MERS-CoV), these three belonging to the class of $\beta$-CoV [4]. The genome sequence of SARS-CoV-2 and SARSCoV homology is about $79 \%$, with interestingly high similarity of the receptor-binding domain (RBD) in spike-protein, confirmed through several analyses, that SARS-CoV-2 uses ACE2 as a receptor, just like SARS-CoV, to recognize the corresponding receptor on the target cell by its $\mathrm{S}$ protein on the surface, then enters into the cell, thereby causing the commencement of infection [1,2]. Structural model assessment, displays SARS-CoV-2 binding ACE2 with more than 10 folds higher affinity than SARS-CoV, much above the required threshold for virus infection [5]. The detailed mechanism, about the SARS-CoV-2 human transmission, interaction, infection, pathological organ damage, still remains unknown, which requires elaborate studies. Considering, the higher binding affinity of SARS-CoV-2 with ACE2, further explains, the larger rapid human transmission capability of SARS-CoV-2 for COVID-19, than by SARS-CoV, that emerged in March 2003 [6].

The current emergence of this novel coronavirus and its heterogeneity, raising serious, alarming threats, while conventional or advanced, even Food and Drug Administration (FDA) approved, medications and diagnostics, are unable to suffice the need for instant management [6]. Also, the unique features identified in the SARS-CoV-2 genome, by gene-level systematic mutational analysis of the genomes, reveal idiosyncratic mutation, including in glycoprotein of the spike surface (A930V (24351C $>$ T)), occurring in some of its strains, as well as the immune epitopes predicted in the genomes, further aggravate the problem [7]. Referring to earlier epidemiological studies, there are three conditions responsible for virus widespread, which are the infection source, transmission route, and susceptibility, with no exception for SARS-CoV$2[1,7]$. Hitherto, four categorized treatments are recognized, Chinese medicine, a western antiviral medicine, immune-enhancement therapy, and convalescent plasma therapy with viral specific plasma globulin [1]. All these deserve larger clinical trials, for determining safety consideration, to be served as standards in clinical practice for COVID-19. SARS-CoV-2 is acutely virulent, transmitted through droplets, close contact, and even touch [1]. By and large, as the exact virus mechanism route remains unclear, no specific drugs could be developed for this novel virus. At present, in the burning situation, it is important to cut-off the virus transmission route, in order to check the progress of this life-threatening disease and protect the people.

Therefore, a coherent strategy is comprehended here to confront, as a convenient means to shield mankind. On the basis of relevant experimental and published data, the perspective presented here brings to light, a transmission chain-breaking effort, via the preventive technique of contamination minimizing a measure of maximized surface coatings with nanosilver endowed self-sterilizing antimicrobial nanocomposite material, consisting antiviral properties, in order to help the public effectively deal with COVID-19. An enormous pool of literature, support the inherent nature of silver nanoparticles (AgNPs) as a usefully potential tool against a variety of viruses, such as Human Immunodeficiency Virus (HIV-1), Nipah virus (NiV), H1N1 Influenza A virus, Respiratory Syncytial Virus (RSV), Herpes Simplex virus 
(HSV), Hepatitis B virus, Poliovirus type-1, Monkey Pox virus, etc. [8-12]. The antiviral activity of nano silver-based silver/chitosan (Ag/CS) nanocomposite coating system, shall be exalted, due to the cohesive action of curcumin [12], that persistently adhere to the nanostructures along with other biomolecules, acting as capping agents, for Curcuma longa rhizome extract aided the formation of AgNPs [13, 14]. Chitosan (CS) biopolymer, neatly alone, exhibits no antiviral activity but provides itself as an excellent matrix phased base for the reinforced phase, facilitating proper dispersion of the AgNPs, while lowering risk concerns of diffusion of the AgNPs into the environment $[10,15,16]$. The relationship between antiviral activity and AgNPs, determined by evaluations suggest that it is inversely proportional to the size, because of spatial hindrance and directly proportional to the concentration of AgNPs, in the manner, the individual virus particles, i.e., virions and nanocomposite interacted [10]. Albeit, the elusive mechanism of action not exclusively investigated, usually the antiviral activity of AgNPs, against several types of viruses, is demonstrated by blocking virus-host cell plasma membrane binding, due to preferential direct AgNP binding to glycoprotein knobs of the viral envelope at the exposed sulfur-bearing residue sites, thereby, inhibiting penetration of virions into the host cells [8-12]. Also, most likely, AgNPs as virucidal agents impair the viral genome, resulting in inactivation of viral replication [9]. AgNPs, even impede host receptor and associated co-receptor dependent virion conformational changes, attachment, merging, and pathogenesis, via viral interaction in cell-free as well as cell-associated states [11]. The lone pair of electrons, present at the sulfhydryl group of the viral envelope, interact with the partial positive charge, developed at the surface of the AgNPs by slight electron drift in the valence band [16].

Curcumin, the polyphenolic compound diferuloylmethane, chemical formula $(1,7-$ bis(4-hydroxy-3-methoxyphenyl)-1,6-heptadiene-3,5-dione), mainly existing in the rhizome part of the herbal plant Curcuma longa L. called turmeric, has enriched medicinal and antimicrobial benefits believed traditionally as well as postulated through explored research investigations and scientific justifications [17]. In-depth, in vitro, and in vivo antiviral assessments of curcumin, has proved its activity in this direction, together with its characteristic behavior of enhancing in manifolds, the inhibitory effect of co-antimicrobial agents in combination, because of synergistic operation [17, 18]. Antiviral action efficacies of the curcumin molecule and derivatives, on a range of emerging enveloped viruses, like the Chikungunya virus (CHIKV), Zika virus (ZIKV), Hepatitis C virus (HCV), Japanese Encephalitis virus (JEV), human papillomavirus (HPV), HIV-1 and HIV-2, HSV-1 and HSV2, RSV, Influenza viruses PR8, H1N1, and H6N1, Dengue virus, etc., and the mechanism paths of deactivation as a virucidal agent are vast [17-22]. Curcumin, being lipophilic molecule alters transfection efficiency of the virus itself from cationic liposomes, interferes with receptor function affecting envelope dynamics and membrane fluidity by conformation changes of viral glycoproteins thus hindering infective essential binding, fusion and replication process [21]. A plethora of reports suggests that curcumin engages itself in antiviral activity by additional diverse mechanisms. Curcumin, for instance, inhibits JEV through the dysregulated ubiquitinproteasome system with an accumulation of the ubiquitinated proteins [19]. HCV being sensitive to an important transcription factor SREBP-1, for sterol synthesis regulation, is inactivated by curcumin via inhibition of the factor [20]. Apart from administering as a potent HIV-1 and HIV-2 protease inhibitor, curcumin directly affects the viral replication machinery of HIV-1 by integrase activity reduction. Specifically, the terminal o-hydroxyl and keto-enol group of curcumin are able to exhibit tight linkage with the acidic binding site formed by 
integrase residues within the catalytic core domain. This is associated with a considerable decrease in the expression of immediate-early (IE) viral genes, caused due to reduced recruitment of RNA polymerase II to IE gene promoters [17, 22]. Nanomaterials, loaded with the curcumin compound, even demonstrated elevated abolition of RSV infection [18].

In the strive to control, the spread of COVID-19, recommended in this article, is a coating of object surfaces having the texture of metallic, ceramic, polymeric, wooden, etc., with antimicrobial Ag/CS nanocomposite, which will not alter the internal physicochemical properties and original functionalities of objects, except for an outer layer of morphological modification, in a move to render self-sterilizing nature externally. The feasible coating system $[15,16]$, with this material of Curcuma longa aided Ag/CS nanocomposite, will possibly restrict the virus SARS-CoV-2 [12] to thrive on object surfaces, predictably reducing touch transmission through surface contamination. An eco-friendly, cost-effective and facile protocol of synthesis for $\mathrm{Ag} / \mathrm{CS}$ nanocomposite, added with the beneficiaries of Curcuma longa rhizome, is proposed here, to facilitate coating over maximum possible exposed surface areas of surrounding objects or parts for the purpose, such as publicly handled door handles, railings, tabletops, switch buttons, touch screens, keyboards, etc., much in common use, towards containment of the viral infection.

\section{Materials and Methods}

\subsection{Materials.}

Chitosan (CS, degree of deacetylation: $79 \%$, molecular mass: 500,000 g/mol), purchased from Sea Foods, Cochin, India; silver nitrate $\left(\mathrm{AgNO}_{3}\right)$ of analytical grade, from Thomas Baker (Chemical) Pvt. Ltd. India; acetic acid glacial (extra pure), from Thomas Baker (Chemical) Pvt. Ltd. India. The rhizome of the perennial herb Curcuma longa (turmeric), collected freshly from the farm. Deionized water, used for the preparation of solutions.

\subsection{Development of Ag/CS nanocomposite coating material.}

A clean piece of fresh turmeric (washed and air-dried), weighing $21 \mathrm{~g}$, was chopped finely and boiled, using $100 \mathrm{ml}$ of sterile deionized water in $500 \mathrm{ml}$ Erlenmeyer flask, for 15 min at $100{ }^{\circ} \mathrm{C}$. The crude extract was filtered using filter paper (Whatman No. 41); thereafter, $10 \mathrm{ml}$ of the filtered turmeric extract (TE) was added to $100 \mathrm{ml}$ of $1 \mathrm{mM}$ colorless $\mathrm{AgNO}_{3}$ solution. The reduction of silver cations $\left(\mathrm{Ag}^{+}\right)$to silver atoms $\left(\mathrm{Ag}^{0}\right)$ and formation of $\mathrm{AgNPs}$, demonstrated by color transformation to brownish-orange and was monitored by measuring the $\mathrm{UV}$-visible spectrum of the reaction mixture $(\mathrm{RM})$ having $\left(\mathrm{AgNO}_{3}\right.$ solution $\left.+\mathrm{TE}\right)$. After $24 \mathrm{~h}$ of complete stabilization at room temperature (RT), RM was centrifuged at 12,000 rpm for 15 min, and the residue (AgNP pellet) was re-dispersed in distilled water and re-centrifuged. This procedure was repeated to isolate AgNPs from loosely bound plant proteins, other bio-organic compounds, and phytochemicals present $[13,14]$. The remnant pellet was reinforced in $15 \mathrm{ml}$ of matrix, CS solution [2 \% (w/v) in 1\% (v/v) acetic acid], at (1:5) ratio and sonicated for 10 min. Finally, Ag/CS nanocomposite was prepared to be used for surface coating. The nanomaterial was synthesized following the principles of green chemistry, avoiding harsh reagents that persistently adhere to the surface of nanostructures and are hazardous to be handled as well as to be harnessed. 


\subsection{Coating.}

Coating of surfaces $[15,16]$, conducted by pouring the Ag/CS nanocomposite suspension (solvent casting technique) and air drying, for preliminary studies conferred desirable results (Fig. 1). At the same time, advanced coating techniques may also be adapted, as per requirements. $\mathrm{Ag} / \mathrm{CS}$ nanocomposite thin film was prepared separately, by casting the suspension on a glass slab, drying at RT and thereafter scratching out, for several characterizations.

(A)

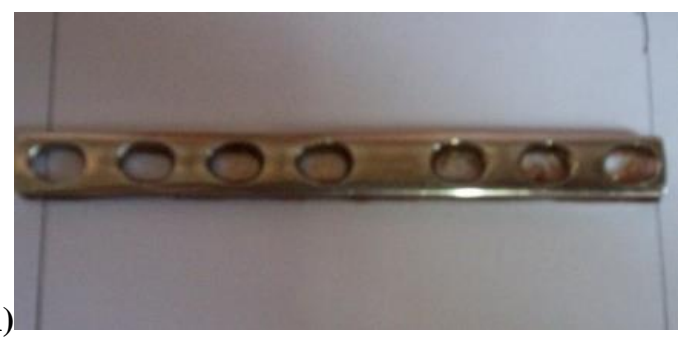

Figure 1. Stainless steel surface coated with Ag/CS nanocomposite: (A) plate; and (B) rod.

\subsection{Characterization.}

The synthesized AgNPs and the developed Ag/CS nanocomposite were characterized performing various techniques and the studies through UV-Visible spectrophotometer, transmission electron microscopy (TEM), selected area electron diffraction (SAED), scanning electron microscopy (SEM), Fourier transformed-infrared (FT-IR) spectroscopy, X-ray diffraction (XRD), are being specified here. UV-Visible spectrophotometer (Shimadzu UV2450) was used to obtain the UV/Visible spectra ranging from 200-800 $\mathrm{nm}$ for governing the phytofabrication of AgNPs and formation of Ag/CS nanocomposite. The identification of shape and size of the particles was with the observations of TEM and SAED images from (TECNAI $20 \mathrm{G}^{2}$ ). Chemical elucidation of the AgNPs and Ag/CS nanocomposite material was enabled with the help of FT-IR spectra recorded between $400-4000 \mathrm{~cm}^{-1}$ using (Varian 3100 ). SEM analysis for morphological features and XRD pattern for structural physical properties of the $\mathrm{Ag} / \mathrm{CS}$ nanocomposite was carried out through (JEOL JXA 8100) and (XRD, Philips, Xpert, $\mathrm{Cu} \mathrm{K \alpha}$ ) at a scanning speed of $3 \% \mathrm{~min}$, respectively.

\section{Results and Discussion}

\subsection{Results.}

TEM images of the AgNPs (Fig. 2A and 2B), illustrated the formation of chiefly spherical along with miscellaneous shaped and irregular sized, distinguish particles, mostly in the nano range. TEM observation indicated no agglomeration or little interparticle interactions, due to the peripheral complexation of capped biomolecules. Turmeric biomolecules, such as reducing sugars and polyphenolic phytochemicals from TE, mediated the reduction of $\mathrm{Ag}^{+}$to $\mathrm{Ag}^{0}$ for the formation of $\mathrm{AgNPs}$, further acted as capping ligands for nanoparticle stabilization. The ring, like approximately circular SAED pattern of the bright spots, denoted the crystalline nature of the nanoparticles (Fig. 2C).

RM undergoing gradual color transformation process from colorless to brownish orange, on complete stabilization showed strong absorbance $\lambda_{\max }$ at $445 \mathrm{~nm}$ with a single surface plasmon resonance (SPR) band revealing the spherical shape of AgNPs and peak being 
in between $430 \mathrm{~nm}$ and $480 \mathrm{~nm}$, characteristic of AgNPs [14, 15], (Fig. 3A). Spectral analysis of the developed $\mathrm{Ag} / \mathrm{CS}$ nanocomposite suspension also exhibited an absorbance peak similar to aqueous suspension counterpart of the AgNPs. The absorption spectrum of TE alone, faired indistinct, not well-defined peak at $\sim 400 \mathrm{~nm}$, of aqueous $\mathrm{AgNO}_{3}$ only solution featured rise at $\sim 220 \mathrm{~nm}$, and CS itself was transparent in the UV-visible region, without any maxima or minima.

(A)

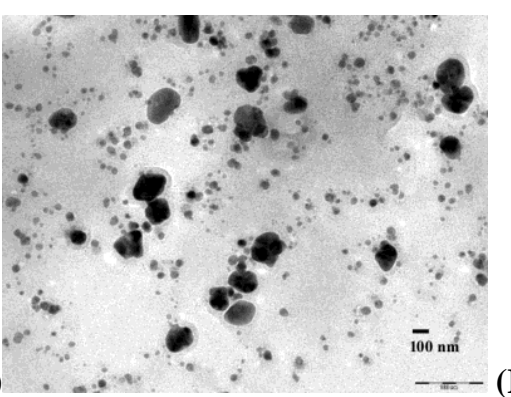

(B)

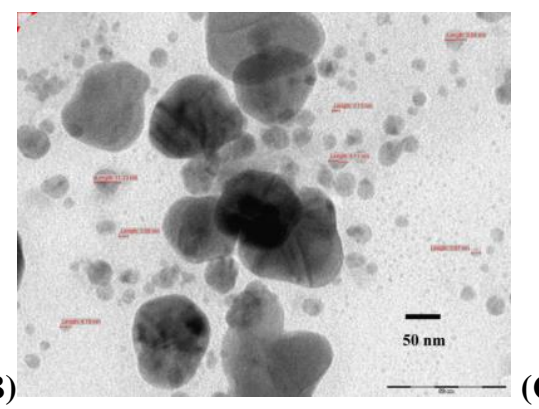

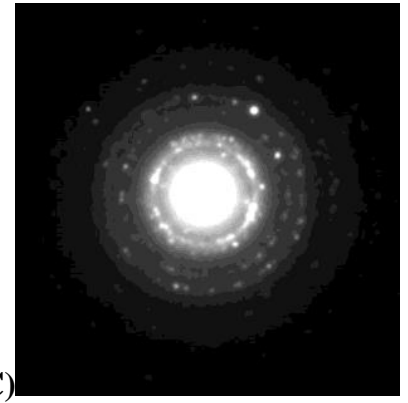

Figure 2. TEM images of AgNPs: (A) and (B) at different magnifications; and (C) SAED pattern.

The FT-IR spectrum of AgNPs (Fig. 3B) elucidated the active chemical species involved in the phytofabrication. The observed large $-\mathrm{OH}$ and $-\mathrm{CH}$ stretches $\left(3417 \mathrm{~cm}^{-1}\right.$ and $2919 \mathrm{~cm}^{-1}$ ) were characteristic of sugar molecules, persistently adhered to the AgNPs. The peak at $\left(1619 \mathrm{~cm}^{-1}\right)$ can be attributed predominantly to the overlapping stretching vibrations of $\mathrm{C}=\mathrm{C}-$ and $-\mathrm{C}=\mathrm{O}$ characters derived from aromatic rings or polyphenolics. The peak at (1384 $\left.\mathrm{cm}^{-1}\right),\left(1077 \mathrm{~cm}^{-1}\right)$ and $\left(616 \mathrm{~cm}^{-1}\right)$ may be ascribed to diverse stretching and bending vibrations of amino acid functional groups. FT-IR study confirmed that plant protein residues, carbohydrates together with polyphenolic molecules as capping moieties were strongly adsorbed on the AgNPs. A spectrum of Ag/CS nanocomposite (Fig. 3B) with intensified bending vibrations in between $1560-1034 \mathrm{~cm}^{-1}$, indicated probable interactions of AgNPs and CS amino groups.
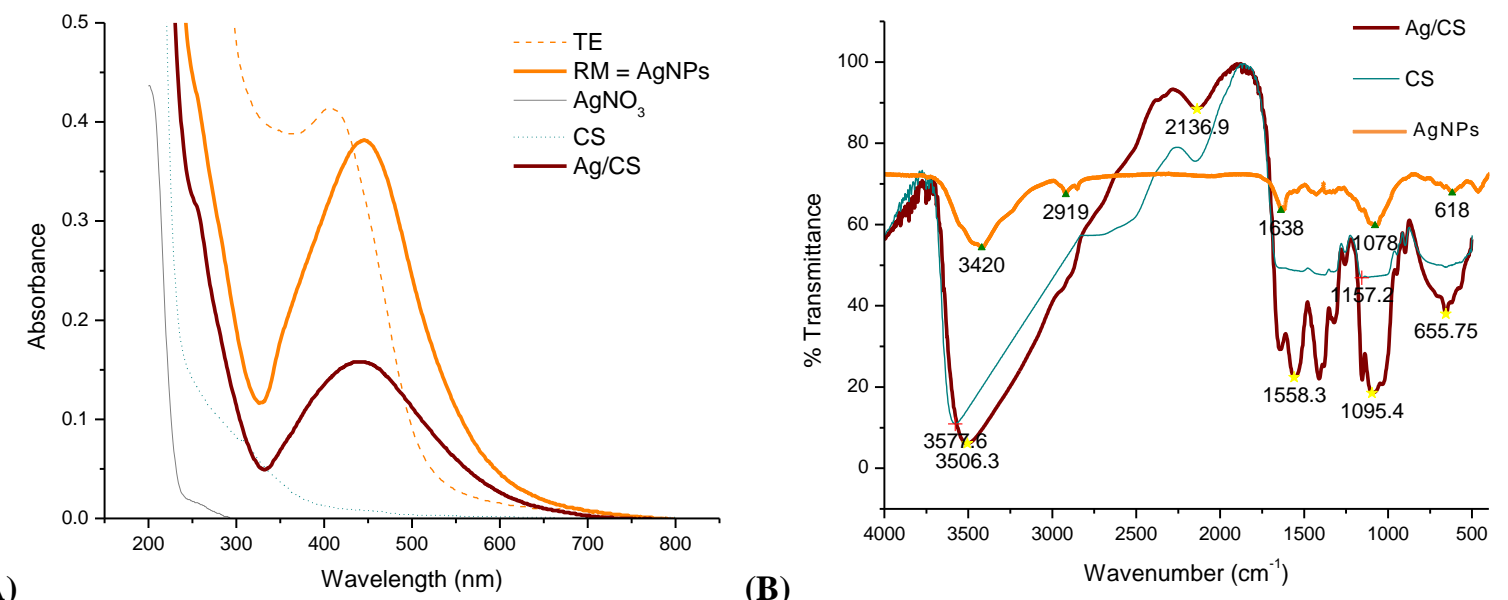

Figu nanocomposite suspension; and (B) FT-IR spectra of AgNP powder, 2\% CS and Ag/CS nanocomposite, films.

SEM micrograph of Ag/CS nanocomposite (Fig. 4A) displayed uniform distribution with proper reinforcement of AgNPs in the CS polymer matrix, rendering smooth surface to the nanomaterial, unfavorable for the sustainability of micro-organisms. XRD pattern of $\mathrm{Ag} / \mathrm{CS}$ nanocomposite (Fig. 4B) defined its amorphous characteristic implicitly. Characterized physicochemical features unambiguously implied, that surface coating with the Ag/CS nanocomposite material, will confer similar results referred to our respective earlier studies 
$[15,16]$ and serve to achieve the concerned present targeted purpose. Mentioning apart, the developed nanomaterial shall also be non-toxic to humans, rather biocompatible in nature, according to our previous reported assessments [16]. Future prospective studies on this nanomaterial coating aspect, focusing the novel coronavirus, will cast more light into the context.

(A)

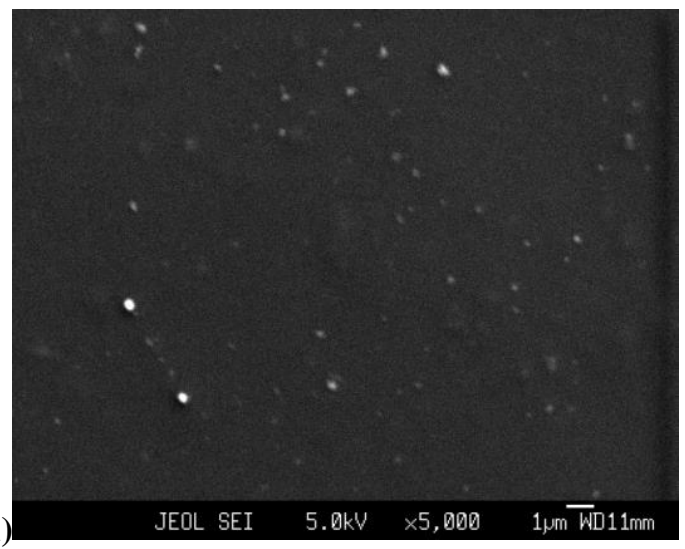

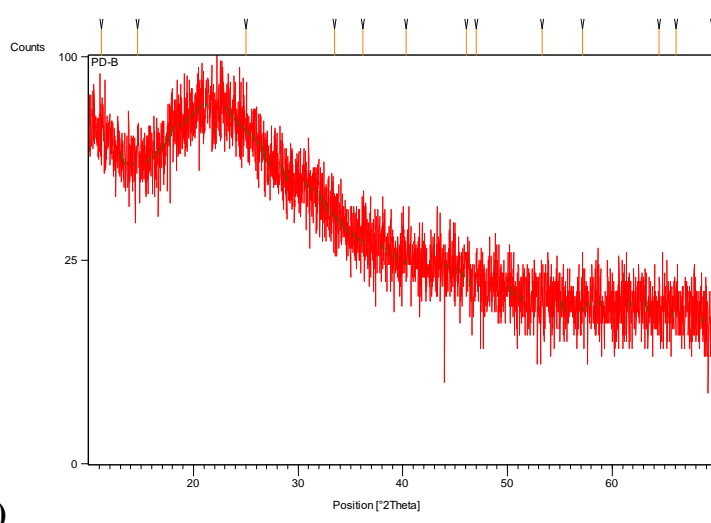

(B)

Figure 4. Ag/CS nanocomposite film: (A) SEM micrograph; and (B) XRD pattern.

\subsection{Discussion.}

Corona regime search for a Holy Grail, to mitigate the escalated menace of COVID-19 pandemic, is continuing with the world baffled in the battle to resist SARS-CoV-2 infection. The ability of the virus to engage its human cellular receptor, enter into the cells, and replicate, being a complex process, throws many opportunities to afford growing antiviral strategies. Findings regarding the interaction of viruses with antiviral nanoparticles, incorporated with different surface chemistry, indicate marked differences in inhibition results, which is explained in terms of dependence upon the capping agents employed in nanoparticle synthesis. Synthetic capping polymers selected, encapsulate the nanoparticle surface, lowering their affinity and inhibitory effect, while phytochemicals involved in reduction and stabilization of nanoparticles, render the nearly free essential binding surfaces, enabling them to interact stronger [23]. Moreover, in our prudent proposition, the AgNPs are embodied with the congruent beneficiaries of bound turmeric biomolecules in traces together with $\mathrm{Ag}$, designed for long-term impact.

\section{Conclusions}

At this juncture, facilitating the proposed, cost-efficient Ag/CS nanocomposite facile surface coating system, over maximum feasible exposed contact areas of surrounding objects and parts, such as railings, door handles, table tops, switch buttons, touch screens, keyboards, etc., mostly which are commonly handled in the community, may bestow huge relief. The same offer stands implied for hospitals, even to medical equipment and devices. The perspective solution shall grant giant rescue from the regular exorbitant and tedious process of repeated disinfection and sterilization. The nanomaterial, casting minimal altered outer appearance to materials, will disallow microbes, definitely including the novel coronavirus, to stick onto their imparted smooth surface modification, comprising self-sterilization, minimizing contamination, and transmission, during this corona fall. 


\section{Funding}

Science and Engineering Research Board (SERB), India, a statutory body of the Department of Science and Technology (DST), Government of India (GI), partially supported through financial assistance [Project File No.: PDF/2017/002264] under the National Post Doctoral Fellowship (N-PDF) scheme.

\section{Acknowledgments}

PD sincerely acknowledges Emeritus Prof. O. N. Srivastava, Department of Physics, Banaras Hindu University, Varanasi, India, for the HR-TEM images.

\section{Conflicts of Interest}

The authors declare no conflict of interest.

\section{References}

1. Wang, L.; Wang, Y.; Ye, D.; Liu, Q. Review of the 2019 novel coronavirus (SARS-CoV-2) based on current evidence. International Journal of Antimicrobial Agents 2020, 55, https://doi.org/10.1016/j.ijantimicag.2020.105948.

2. Qiu, Y.; Zhao, Y.-B.; Wang, Q.; Li, J.-Y.; Zhou, Z.-J.; Liao, C.-H.; Ge, X.-Y. Predicting the angiotensin converting enzyme 2 (ACE2) utilizing capability as the receptor of SARS-CoV-2. Microbes and Infection 2020, 22, 221-225, https://doi.org/10.1016/j.micinf.2020.03.003.

3. Olsen, M.; Cook, S.E.; Huang, V.; Pedersen, N.; Murphy, B.G. Perspectives: potential therapeutic options for SARS-CoV-2 patients based on feline infectious peritonitis strategies: central nervous system invasion and drug coverage. International Journal of Antimicrobial Agents 2020, 55, https://doi.org/10.1016/j.ijantimicag.2020.105964.

4. Zhu, N.; Zhang, D.; Wang, W.; Li, X.; Yang, B.; Song, J.; Zhao, X.; Huang, B.; Shi, W.; Lu, R.; Niu, P.; Zhan, F.; Ma, X.; Wang, D.; Xu, W.; Wu, G.; Gao, G.F.; Tan, W. A Novel Coronavirus from Patients with Pneumonia in China, 2019. New England Journal of Medicine 2020, 382, 727-733, https://doi.org/10.1056/NEJMoa2001017.

5. Wrapp, D.; Wang, N.; Corbett, K.S.; Goldsmith, J.A.; Hsieh, C.L.; Abiona, O.; Graham, B.S.; McLellan, J.S. Cryo-EM structure of the 2019-nCoV spike in the prefusion conformation. Science 2020, 367, 12601263, https://doi.org/10.1126/science.abb2507.

6. Devaux, C.A.; Rolain, J.-M.; Colson, P.; Raoult, D. New insights on the antiviral effects of chloroquine against coronavirus: what to expect for COVID-19? International Journal of Antimicrobial Agents 2020, 55, https://doi.org/10.1016/j.ijantimicag.2020.105938.

7. Lai, C.-C.; Shih, T.-P.; Ko, W.-C.; Tang, H.-J.; Hsueh, P.-R. Severe acute respiratory syndrome coronavirus 2 (SARS-CoV-2) and coronavirus disease-2019 (COVID-19): The epidemic and the challenges. International Journal of Antimicrobial Agents 2020, 55, https://doi.org/10.1016/j.ijantimicag.2020.105924.

8. Elechiguerra, J.L.; Burt, J.L.; Morones, J.R.; Camacho-Bragado, A.; Gao, X.; Lara, H.H.; Yacaman, M.J. Interaction of silver nanoparticles with HIV-1. Journal of Nanobiotechnology 2005, 3, https://doi.org/10.1186/1477-3155-3-6.

9. Galdiero, S.; Falanga, A.; Vitiello, M.; Cantisani, M.; Marra, V.; Galdiero, M. Silver Nanoparticles as Potential Antiviral Agents. Molecules 2011, 16, 8894-8918, https://doi.org/10.3390/molecules16108894.

10. Mori, Y.; Ono, T.; Miyahira, Y.; Nguyen, V.Q.; Matsui, T.; Ishihara, M. Antiviral activity of silver nanoparticle/chitosan composites against H1N1 influenza A virus. Nanoscale research letters 2013, 8, 93, https://doi.org/10.1186/1556-276X-8-93.

11. Kerry, R.G.; Malik, S.; Redda, Y.T.; Sahoo, S.; Patra, J.K.; Majhi, S. Nano-based approach to combat emerging viral (NIPAH virus) infection. Nanomedicine: Nanotechnology, Biology and Medicine 2019, 18, 196-220, https://doi.org/10.1016/j.nano.2019.03.004.

12. Yang, X.X.; Li, C.M.; Huang, C.Z. Curcumin modified silver nanoparticles for highly efficient inhibition of respiratory syncytial virus infection. Nanoscale 2016, 8, 3040-3048, https://doi.org/10.1039/C5NR07918G.

13. Dwivedi, P.; Narvi, S.; Tewari, R. Natural route to a novel nanocomposite biomaterial: an embodiment for the abatement of implant associated infection. International Conference on Nanoscience, Technology and Societal Implications 2011. doi:10.1109/NSTSI.2011.6111809.

14. Dwivedi, P.; Narvi, S.S.; Tewari, R.P. Phytofabrication characterization and comparative analysis of Ag nanoparticles by diverse biochemicals from Elaeocarpus ganitrus Roxb., Terminalia arjuna Roxb., 
Pseudotsuga menzietii, Prosopis spicigera, Ficus religiosa, Ocimum sanctum, Curcuma longa. Industrial Crops and Products 2014, 54, 22-31, http://dx.doi.org/10.1016/j.indcrop.2013.12.050.

15. Dwivedi, P.; Narvi, S.; Tewari, R. Potentiality of the plant Pseudotsuga menzietii to combat implant-related infection in the nanoregime. Int. J. of Biomedical Nanoscience and Nanotechnology 2012, 2, 187-206, https://doi.org/10.1504/IJBNN.2012.051217.

16. Dwivedi, P.; Narvi, S.S.; Tewari, R.P. Coating Made from Pseudotsuga menziesii Phytosynthesized Silver Nanoparticles is Efficient Against Staphylococcus aureus Biofilm Formation. Nano LIFE 2015, 5, https://doi.org/10.1142/S1793984415400061.

17. Zorofchian Moghadamtousi, S.; Abdul Kadir, H.; Hassandarvish, P.; Tajik, H.; Abubakar, S.; Zandi, K. A Review on Antibacterial, Antiviral, and Antifungal Activity of Curcumin. BioMed Research International 2014, 2014, http://dx.doi.org/10.1155/2014/186864.

18. Yang, X.X.; Li, C.M.; Li, Y.F.; Wang, J.; Huang, C.Z. Synergistic antiviral effect of curcumin functionalized graphene oxide against respiratory syncytial virus infection. Nanoscale 2017, 9, 16086-16092, https://doi.org/10.1039/c7nr06520e.

19. Dutta, K.; Ghosh, D.; Basu, A. Curcumin Protects Neuronal Cells from Japanese Encephalitis VirusMediated Cell Death and also Inhibits Infective Viral Particle Formation by Dysregulation of UbiquitinProteasome System. Journal of Neuroimmune Pharmacology 2009, 4, 328-337, http://dx.doi.org/10.1007/s11481-009-9158-2.

20. Kim, K.; Kim, K.H.; Kim, H.Y.; Cho, H.K.; Sakamoto, N.; Cheong, J. Curcumin inhibits hepatitis C virus replication via suppressing the Akt-SREBP-1 pathway. FEBS Letters 2010, 584, 707-712, http://dx.doi.org/10.1016/j.febslet.2009.12.019.

21. Mounce, B.C.; Cesaro, T.; Carrau, L.; Vallet, T.; Vignuzzi, M. Curcumin inhibits Zika and chikungunya virus infection by inhibiting cell binding. Antiviral Res 2017, 142, 148-157, https://doi.org/10.1016/j.antiviral.2017.03.014.

22. Praditya, D.; Kirchhoff, L.; Brüning, J.; Rachmawati, H.; Steinmann, J.; Steinmann, E. Anti-infective Properties of the Golden Spice Curcumin. 2019, 10, https://doi.org/10.3389/fmicb.2019.00912.

23. Dwivedi, P.; Tiwary, D.; Mishra, P.K.; Chakraborty, J.P. MgO/CaO nanostructures fabricated from trimmedoff human finger and toe nails. Nano-Structures \& Nano-Objects 2020, 22, 100485, https://doi.org/10.1016/j.nanoso.2020.100485. 